\title{
The O-type eclipsing binary SZ Camelopardalis revisited ${ }^{\star}$ (Research Note)
}

\author{
P. Mayer ${ }^{1}$, H. Drechsel ${ }^{2}$, J. Kubát ${ }^{3}$, and M. Šlechta ${ }^{3}$ \\ ${ }^{1}$ Astronomical Institute of the Charles University, Faculty of Mathematics and Physics, V Holešovičkách 2, 18000 Praha 8, \\ Czech Republic \\ e-mail: mayer@cesnet.cz \\ 2 Dr. Remeis-Sternwarte, Astronomisches Institut der Universität Erlangen-Nürnberg, Sternwartstraße 7, 96049 Bamberg, Germany \\ 3 Astronomical Institute, Academy of Sciences of the Czech Republic, Fričova 298, 25165 Ondřejov, Czech Republic
}

Received 14 October 2009 / Accepted 24 October 2010

\section{ABSTRACT}

\begin{abstract}
We analyse new spectra of the multiple system SZ Cam because previous studies found different values of the primary radial velocity amplitude. The older solutions of light curves also have different ratios of secondary to primary luminosity as inferred from the observed equivalent widths of spectral lines. We therefore reanalyse the light curves of the eclipsing pair. Only the light curve derived by Wesselink has a solution that agrees with the observed equivalent width ratio. The resulting parameters of the binary are discussed. Masses of $M_{1}=16.6$ and $M_{2}=11.9 M_{\odot}$, and radii $R_{1}=9.4$ and $R_{2}=5.4 R_{\odot}$ are derived. We point out that radial velocities measured with the CCF method can be misleading when the method is applied to multiple systems with complex line blends. New radial velocities are also obtained for the visual component ADS 2984 A (HD 25639).
\end{abstract}

Key words. binaries: eclipsing - stars: early-type - stars: fundamental parameters - stars: individual: SZ Cam stars: individual: ADS 2984 A

\section{Introduction}

The early-type eclipsing binary SZ Cam (HD 25638) has been the subject of several studies in recent years. The binary is a member of the open cluster NGC 1502 and is also the northern component of the visual binary ADS 2984. With a combined spectral type of $\mathrm{O} 9.5$ and detached components, the binary is a potentially important source of absolute parameters of massive stars. However, the star was found to be a triple system - or, more specifically, a system consisting of two close binaries (Mayer et al. 1994). The third contributor to the light makes the light curve solution difficult, and also causes problems in the analysis of the complex spectra.

The third body in SZ Cam manifests itself in various ways: as a third component in the spectral line profiles, by the presence of the light time effect, and by additive light in the light curves (Lorenz et al. 1998, hereafter LMD). The third body was also found independently by speckle interferometry (Mason et al. 1998). A new speckle measurement was published by Gorda et al. (2007), and the magnitude difference was given by Balega et al. (2007) as $0.95 \pm 0.02$ at $\lambda 535 \mathrm{~nm}$. In the Washington Double Star Catalog ${ }^{1}$, SZ Cam is referred to as component Ea, and the speckle component as Eb.

In addition to these studies, the star was analysed by Harries et al. (1998, hereafter HHH), Gorda (2000, 2002, 2008), and Michalska et al. (2007). HHH found some basic parameters of

* Based on spectral observations from the Ondřejov Observatory and on observations collected at the German-Spanish Astronomical Centre, Calar Alto, operated by the Max-Planck-Institute für Astronomy, Heidelberg, jointly with the Instituto de Astrofisica de Andalusia.

1 http://ad.navy. usno.mil/wds the system, namely the semiamplitude $K_{1}$, which differs from those published by LMD.

We collected new spectra with the $2 \mathrm{~m}$ telescope at the Ondřejov Observatory and also derived new solutions of the light curves. Based on these data, we expect to resolve the discrepancy between $\mathrm{HHH}$ and LMD. The already published results and our new results are listed in Table 1 . Note that the radial velocity (RV) semiamplitudes $K_{1}$ found by Michalska et al. (2007) and by Gorda (2008) are close to those of LMD. In this paper, the linear ephemeris given by Gorda et al. (2007) is used:

Primary minimum $=$ HJD 2453676.1628 +2.6984222.

\section{Spectroscopy}

The spectra analysed by LMD were obtained with the $2.2 \mathrm{~m}$ telescope at the Calar Alto Astronomical Centre (hereafter CA). New spectra for SZ Cam were recorded with the coudé spectrograph at the $2 \mathrm{~m}$ telescope of the Ondřejov Observatory. The region between $627 \mathrm{~nm}$ and $678 \mathrm{~nm}$ is covered by 46 spectra with a two-pixel resolution of 12700 and a typical signal-to-noise ratio $(\mathrm{S} / \mathrm{N})$ of $\approx 200$. There are also two echelle spectra covering the region from $400 \mathrm{~nm}$ to $670 \mathrm{~nm}$ publicly available at the ELODIE archive (Moultaka et al. 2004). The spectral region of the He I 6678 A line in two Ondrejov spectra taken at opposite quadrature phases is shown in Fig. 1. The relatively narrow and strong third-body line is clearly visible in the centre of the blend. Owing to the strong reddening $\left(E_{B-V}=0.68\right)$, several DIBs are present: a stronger one at $6672.0 \mathrm{~A}$ with equivalent width $(E W)$ of about $0.02 \mathrm{~A}$ and several other weaker ones at 6681.1, 6684.4, and 6689.0 A (the wavelengths of these DIBs were measured in 
Table 1. The published parameters of the RV curve (values in $\mathrm{km} \mathrm{s}^{-1}$ ).

\begin{tabular}{lllllrll}
\hline \hline Source & $K_{1}$ & m.e. & $K_{2}$ & m.e. & $V_{\gamma}$ & m.e. & Method \\
\hline Harries et al. (1998) & 225.8 & 3.8 & 259.1 & 4.0 & -12.0 & 3.0 & CCF method \\
Lorenz et al. (1998) & 180.2 & 2.0 & 261.2 & 3.8 & -2.9 & 1.6 & MIDAS Gaussian fitting \\
Gorda et al. (2007) & 181.6 & & 268.2 & & & & KOREL applied to H $\alpha$ \\
Gorda (2008) & 192.0 & 2.6 & 266.4 & 2.5 & -10.6 & 2.0 & Gaussian fitting \\
This paper & 189.4 & 1.4 & 264.1 & 2.5 & -2.3 & & Gaussian fitting \\
\hline
\end{tabular}

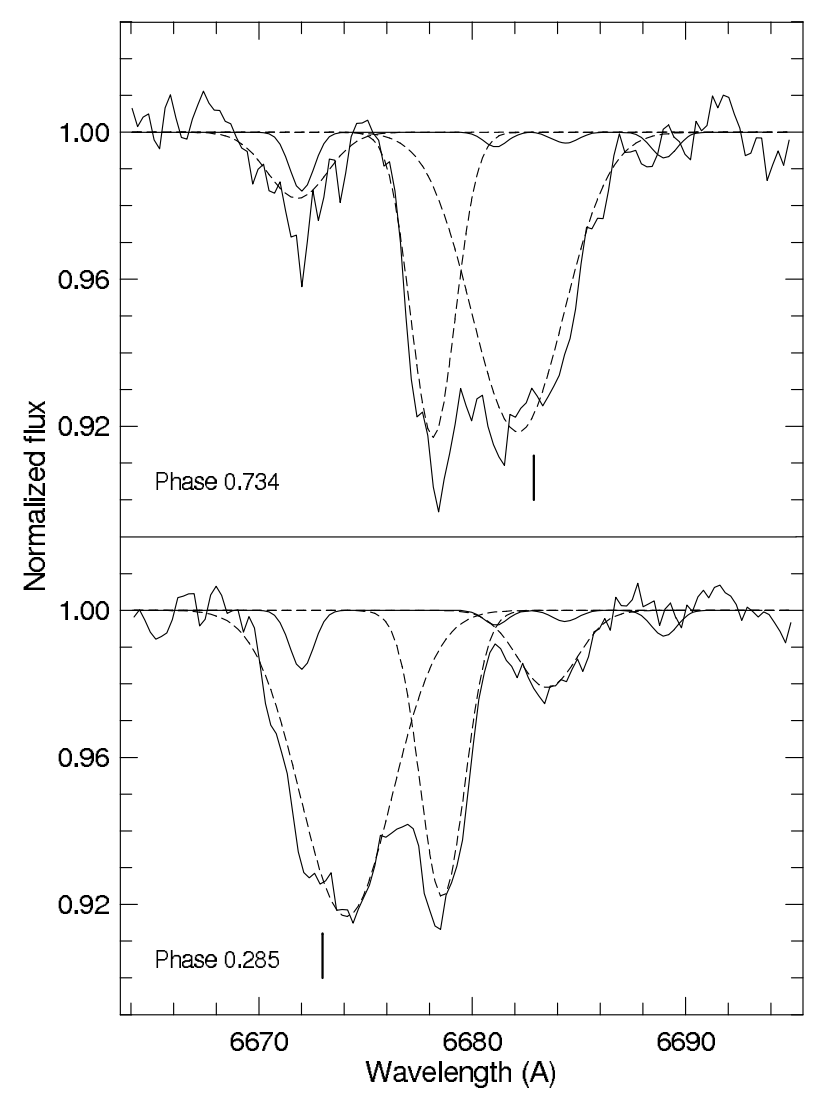

Fig. 1. Profiles of the line He I 6678 A taken close to opposite quadratures, at phases 0.285 and 0.734 . The dashed lines represent the Gaussian fits of all three components of the system (the wide profile - primary, the shallow one - secondary, the narrow one - tertiary). The short vertical lines show the expected primary line positions according to the HHH solution. Four superimposed DIBs are also indicated.

our spectra $)^{2}$. Two short vertical lines mark the primary radial velocities (RVs) as they follow from the RV curve solution by HHH. These RVs are clearly incompatible with our spectra, and the large value of $K_{1}$ published by HHH is not supported.

We fitted the line profiles by Gaussians. The resulting RVs are listed in Table 2. Owing to the complicated structure of the profile, reliable results can only be expected from spectra taken near quadratures, when the secondary line is separated. At phases around 0.75 , the secondary component is blended with the DIB at $6672 \mathrm{~A}$. Close to the He I line, there is the He II line $6683 \mathrm{~A}$. We did not detect any contribution of this line, although in other stars of nearly identical spectral type - but probably of higher luminosity - the He II line plays a role (e.g. at $\delta$ Ori).

\footnotetext{
2 At conjunctions, the DIBs 6672.0 A and 6689.0 A are not blended with the binary lines and can be measured relatively well. The other two DIBs are always superimposed on stellar profiles; their parameters were determined by optimizing the multiple Gaussian fit of the composite profile.
}

Table 2. New radial velocities.

\begin{tabular}{|c|c|c|c|c|c|}
\hline $\begin{array}{l}\text { HJD } \\
-2400000 \\
\end{array}$ & $\begin{array}{l}\text { Phase } \\
\text { ecl. }\end{array}$ & $\begin{array}{l}\text { RV } \\
\text { pri. }\end{array}$ & $\begin{array}{l}\text { RV } \\
\text { sec. }\end{array}$ & $\begin{array}{l}\text { Phase } \\
\text { third }\end{array}$ & $\begin{array}{r}\mathrm{RV} \\
\text { third }\end{array}$ \\
\hline 53706.2888 & 0.1625 & -166.2 & 216.2 & 0.0757 & -18.5 \\
\hline 53706.3075 & 0.1694 & -164.9 & 222.1 & 0.0824 & -26.7 \\
\hline 53706.3777 & 0.1954 & -175.9 & 249.7 & 0.1074 & -21.2 \\
\hline 53706.4290 & 0.2145 & -191.4 & 233.3 & 0.1259 & -17.7 \\
\hline 53713.1984 & 0.7231 & 198.4 & -261.0 & 0.5460 & 34.0 \\
\hline 53713.2673 & 0.7487 & 179.2 & -261.8 & 0.5707 & 30.2 \\
\hline 53743.2557 & 0.8619 & 152.3 & -181.9 & 0.2924 & 14.8 \\
\hline 53744.2390 & 0.2263 & -182.5 & 242.3 & 0.6439 & 21.4 \\
\hline 53747.3102 & 0.3645 & -140.4 & 192.9 & 0.7419 & -1.9 \\
\hline 53817.4299 & 0.3500 & -174.1 & 197.2 & 0.8118 & -20.9 \\
\hline 53818.4625 & 0.7325 & 179.2 & -285.2 & 0.1810 & 0.5 \\
\hline 53832.4657 & 0.9220 & 108.0 & -131.7 & 0.1875 & -0.7 \\
\hline 53938.4909 & 0.2135 & -182.6 & 242.7 & 0.0946 & -19.7 \\
\hline 53938.5082 & 0.2200 & -182.0 & 237.8 & 0.1007 & -18.1 \\
\hline 53938.5465 & 0.2341 & -182.4 & 250.4 & 0.1144 & -15.4 \\
\hline 53938.5810 & 0.2469 & -183.7 & 262.5 & 0.1267 & -15.0 \\
\hline 54014.3879 & 0.3400 & -163.3 & 200.5 & 0.2299 & -9.1 \\
\hline 54014.4173 & 0.3510 & -170.4 & 203.2 & 0.2404 & -6.4 \\
\hline 54020.5757 & 0.6331 & 152.5 & -207.1 & 0.4421 & 25.7 \\
\hline 54028.3257 & 0.5051 & -42.8 & 68.6 & 0.2130 & -29.3 \\
\hline 54028.3496 & 0.5140 & -44.1 & 68.5 & 0.2216 & -25.6 \\
\hline 54028.4000 & 0.5327 & -21.7 & 78.4 & 0.2396 & -27.6 \\
\hline 54085.4032 & 0.6573 & 163.9 & -216.5 & 0.6199 & 23.7 \\
\hline 54085.4771 & 0.6847 & 178.2 & -241.6 & 0.6462 & 25.4 \\
\hline 54085.5942 & 0.7281 & 185.7 & -267.2 & 0.6881 & 15.6 \\
\hline 54085.6617 & 0.7532 & 184.4 & -286.1 & 0.7123 & 12.2 \\
\hline 54085.7320 & 0.7792 & 192.0 & -276.2 & 0.7374 & 12.2 \\
\hline 54097.2111 & 0.0332 & -51.2 & 51.5 & 0.8416 & -16.7 \\
\hline 54097.2940 & 0.0638 & -94.7 & 87.4 & 0.8712 & -17.2 \\
\hline 54097.3726 & 0.0930 & -107.9 & 118.8 & 0.8993 & -15.0 \\
\hline 54097.4929 & 0.1376 & -155.8 & 213.1 & 0.9423 & -28.5 \\
\hline 54097.6107 & 0.1813 & -180.6 & 221.6 & 0.9844 & -31.8 \\
\hline 54097.7207 & 0.2220 & -184.4 & 253.0 & 0.0237 & -26.8 \\
\hline 54166.4347 & 0.6865 & 188.5 & -286.5 & 0.5909 & 30.5 \\
\hline 54166.4617 & 0.6965 & 188.8 & -269.9 & 0.6006 & 29.9 \\
\hline 54166.5121 & 0.7152 & 186.4 & -277.1 & 0.6187 & 27.3 \\
\hline 54166.5434 & 0.7268 & 189.1 & -278.0 & 0.6298 & 27.3 \\
\hline 54533.4269 & 0.6890 & 185.6 & -240.3 & 0.8011 & 2.6 \\
\hline 54533.4716 & 0.7056 & 185.9 & -263.6 & 0.8171 & -3.3 \\
\hline 54581.3352 & 0.4431 & -85.4 & 109.0 & 0.9297 & -15.5 \\
\hline 54590.3348 & 0.7785 & 188.2 & -245.7 & 0.1473 & -2.0 \\
\hline 54594.4061 & 0.2874 & -182.4 & 240.9 & 0.6029 & 20.4 \\
\hline 54620.3787 & 0.9124 & 114.8 & -142.4 & 0.8889 & -21.0 \\
\hline $54798.6220^{a}$ & 0.9668 & 35.8 & 2.6 & 0.6160 & 29.9 \\
\hline 54932.4342 & 0.5562 & 63.2 & -94.4 & 0.4575 & 22.1 \\
\hline 55060.5181 & 0.0222 & -55.2 & 57.3 & 0.2512 & 4.4 \\
\hline
\end{tabular}

Notes. ${ }^{(a)}$ both SZ Cam and ADS 2984 A exposed in same frame.

We also refitted the CA spectra using an identical procedure. The RVs of the eclipsing components are shown in Fig. 2. The solution of the RV curves is listed in Table 3. Only RVs obtained using the line He I $6678 \mathrm{~A}$ from spectra taken in Ondrejov and 
Table 3. Solution of the radial velocity curve of the eclipsing pair.

\begin{tabular}{lrrrr}
\hline \hline & \multicolumn{2}{c}{ Individual $V_{\gamma}$} & \multicolumn{2}{c}{ Common $V_{\gamma}$} \\
\hline Parameter & Primary & Secondary & Primary & Secondary \\
\hline$K\left(\mathrm{~km} \mathrm{~s}^{-1}\right)$ & $189.3 \pm 1.2$ & $263.4 \pm 1.8$ & $189.4 \pm 1.4$ & $264.1 \pm 2.5$ \\
$V_{\gamma}\left(\mathrm{km} \mathrm{s}^{-1}\right)$ & $1.8 \pm 1.0$ & $-10.4 \pm 1.6$ & -2.3 & -2.3 \\
Average $O-C\left(\mathrm{~km} \mathrm{~s}^{-1}\right)$ & 5.1 & 11.5 & 7.0 & 12.6 \\
$A \sin i\left(R_{\odot}\right)$ & $10.09 \pm 0.09$ & $14.04 \pm 0.14$ & $10.10 \pm 0.10$ & $14.08 \pm 0.1$ \\
\hline
\end{tabular}

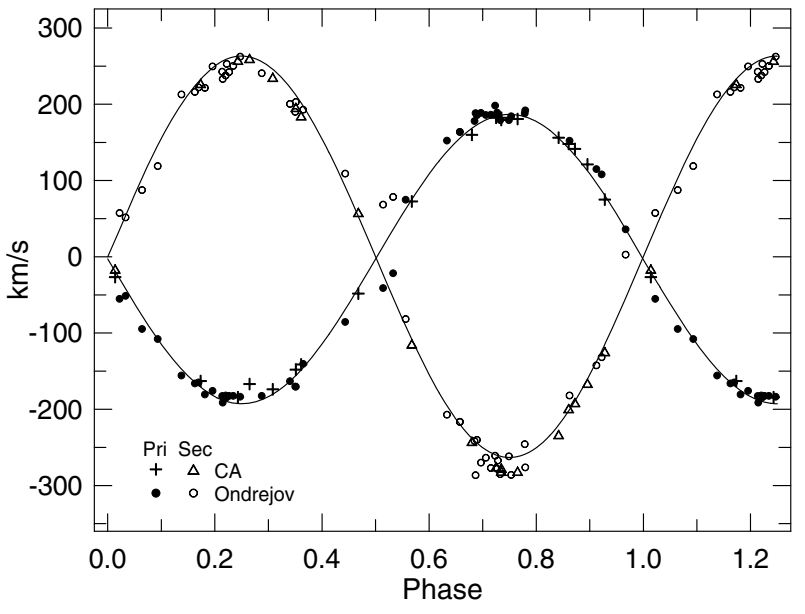

Fig. 2. Radial velocities of the eclipsing pair (the curves represent the solution with common $V_{\gamma}$ ).

Calar Alto were used; velocities from phases closer than 0.08 to both conjunctions were not considered. In the covered spectral range, only the $\mathrm{H} \alpha$ line is present in addition to the $6678 \mathrm{~A}$ line. However, owing to its large width and disturbance by atmospheric lines it is not suitable for Gaussian fitting.

In Table 2, RVs of the third lines are also contained. Since our new solution of the third-body orbit does not differ considerably from that in LMD, we do not discuss these RVs here. Several spectra of the component ADS 2984 A were also obtained, and the RVs are given in Table 4. As the lines are wide, the resulting RVs are of low accuracy and the period of this probable binary has not yet been able to be determined.

Among the existing classifications of SZ Cam, probably the most reliable is the one according to Budding (1978): O 9.5 V. This is the combined spectral type. Following the scheme of Conti \& Alschuler (1971), we confirmed this by comparing the lines 4471/4541 A (spectral type) and 4089/4143 A (luminosity) in the ELODIE spectra. However, a more detailed spectral type classification of the secondary and tertiary components using the ELODIE spectra is not possible due to weakness of the components' spectral features. These components are probably main-sequence stars, therefore their types and temperatures follow from their magnitude differences from the primary. Both should be later than the primary, so the primary might be somewhat earlier than the type of the integral spectrum. Its luminosity is also higher, since the radius is larger than the radius of a MS member as shown in Sect. 3; its $\log g$ is 3.7, a value typical of the luminosity class IV (Martins et al. 2005). Although in stars of earlier spectral type the He II lines are usually observed, this is not so in this case because the strengths of both the $6406 \mathrm{~A}$ and 6683 A lines strongly depend on $\log g$. According to Lanz $\&$ Hubeny (2003), the lines are absent for $\log g$ larger than 3.25 at $T_{\text {eff }}=30500 \mathrm{~K}$.
Table 4. Radial velocities of ADS 2984 A.

\begin{tabular}{lrlr}
\hline \hline $\begin{array}{l}\text { HJD } \\
-2400000\end{array}$ & $\begin{array}{r}\text { RV } \\
\mathrm{km} \mathrm{s}^{-1}\end{array}$ & $\begin{array}{l}\text { HJD } \\
-2400000\end{array}$ & $\begin{array}{r}\text { RV } \\
\mathrm{km} \mathrm{s}^{-1}\end{array}$ \\
\hline 53666.3657 & 23.2 & 54166.5829 & -31.1 \\
53670.3298 & 23.2 & 54170.4173 & -8.4 \\
$53680.6565^{a}$ & 10.0 & 54523.3472 & 20.1 \\
53797.4386 & 12.4 & 54593.3397 & 18.3 \\
53818.4075 & -47.3 & $54798.6220^{b}$ & -17.2 \\
53938.5452 & -10.5 & 55060.5444 & 1.7 \\
53947.4385 & 13.3 & 55074.4146 & -51.3 \\
54028.4483 & -26.0 & 55075.6340 & -49.5 \\
54166.4878 & -15.6 & & \\
\hline
\end{tabular}

Notes. ${ }^{(a)}$ spectrum from the ELODIE archive; ${ }^{(b)}$ both SZ Cam and ADS 2984 A exposed in same frame.

\section{New solutions of light curves}

In the following, we consider several SZ Cam light curves:

- a photographic curve by Wesselink (1941), the quality of which is comparable to photoelectric curves, since it is based on more than 12000 measurements;

- UBV curves by Kitamura \& Yamasaki (1972, $K-Y$ curves);

- two curves obtained with intermediate wide filters centred on 4720 and 5720 A by Chochol (1980);

- UBVR curves by Gorda (2000) obtained with an area scanner, and published in a graphical form only.

Owing to the close vicinity of ADS 2984 A and E, their Hipparcos photometry (ESA 1997) is unhelpful.

These light curves differ for two possible reasons:

- the light curve is variable, or

- the measurements are not reliable.

The variability of stars of the given parameters is not common. There are no indications that the magnitude in maximum changes as it would be in the case of temperature or dimension changes. This explanation is therefore improbable.

Observing SZ Cam with a standard PMT photometer was not easy, because of the close vicinity of the visual component. The $K-Y$ and Chochol curves differ, each other and from the photographic curve. Gorda shows that there were deviations from the average curve in some nights. However, more data would be needed to verify the changes; the variability of the comparison star might be also the reason. The photographic curve was collected over more than 6 years, which had the advantage of smoothing the eventual short-time light curve variability.

A set of solutions is presented in Table 5. In our solutions $T_{1}=30500 \mathrm{~K}$ was assumed. A value close to this was already used by $\mathrm{HHH}$, and according to newer temperature scales (e.g. Massey et al. 2005) it is a reasonable value for the spectral type of the primary component. 
Table 5. Solution of various light curves.

\begin{tabular}{lccccc}
\hline \hline Parameter & $\mathrm{HHH}^{a}$ & $\begin{array}{c}\text { Chochol } \\
\text { curves }^{c}\end{array}$ & LMD $^{b}$ & $\begin{array}{c}\text { Chochol } \\
\text { curves }^{d}\end{array}$ & Wesselink \\
\hline$L_{3}{ }^{e}$ & $27.4 \%^{f}$ & $24.9 \%$ & $31.0 \% \pm 1.5$ & $24.7 \% \pm 3.8$ & $30.9 \% \pm 1.8$ \\
$q$ & 0.871 & 0.871 & $0.704 \pm 0.015$ & $0.710 \pm 0.064$ & 0.718 \\
$T_{1}(\mathrm{~K})$ & 29725 & 30500 & 30500 & 30500 & 30500 \\
$T_{2}(\mathrm{~K})$ & 27183 & 27200 & $25370 \pm 230^{g}$ & $27270 \pm 650$ & $25300 \pm 740$ \\
$i(\mathrm{deg})$ & $76.9 \pm 0.4$ & 76.7 & $77.0 \pm 0.6$ & $77.0 \pm 1.8$ & $79.7 \pm 1.3$ \\
$L_{2} / L_{1}$ in $V$ & $0.773^{f}$ & 0.536 & $0.422 \pm 0.031$ & $0.492 \pm 0.054$ & $0.262 \pm 0.029$ \\
$r_{1} / A$ & $0.319 \pm 0.010$ & 0.344 & $0.380 \pm 0.030$ & $0.353 \pm 0.016$ & $0.379 \pm 0.004$ \\
$r_{2} / A$ & $0.297 \pm 0.012$ & 0.270 & $0.280 \pm 0.030$ & $0.265 \pm 0.021$ & $0.217 \pm 0.017$ \\
\hline
\end{tabular}

Notes. ${ }^{(a)}$ Solution by HHH; they used the two-filter data by Chochol (1980). ${ }^{(b)}$ LMD WD solution of Kitamura \& Yamasaki $U B V$ curves. ${ }^{(c)}$ New solution of Chochol's curves with WD code and the mass-ratio $q$ used by HHH. ${ }^{(d)}$ New solution of Chochol's curves with our $q .{ }^{(e)}$ Assuming $L_{1}+L_{2}+L_{3}=1 .{ }^{(f)}$ Calculated from absolute visual magnitudes in HHH Table $6 .{ }^{(g)}$ Recalculated for $T_{1}=30500 \mathrm{~K}$.

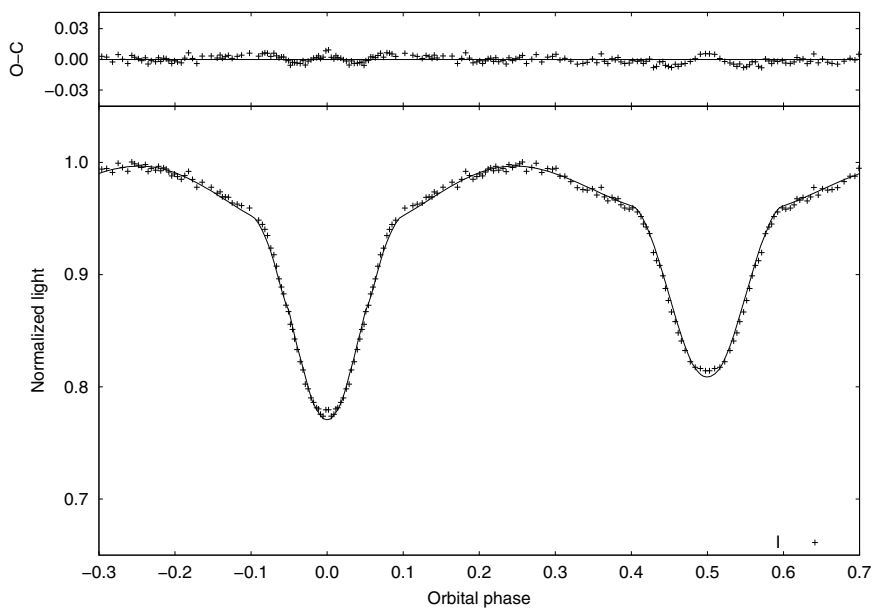

Fig. 3. Solution of the Wesselink light curve with WD code. In the top panel, the differences "measurements minus theoretical curve" are plotted.

Naturally, as the curves differ, their solutions differ too. The largest differences are among the solutions with $q=0.71$ and the HHH solution, with $q=0.871$. We also solved the Chochol curves with $q=0.871$. The adopted codes (LIGHT by HHH and WD by ourselves) are similar, hence the probable reason for the different results is that the Chochol curves constrain the system parameters only weakly. We also found that the scatter of normal points as published by Chochol is about twice as large as that of the $U B V$ data used in LMD, and that the coverage of both minima is superior in the $U B V$ data. When solving the Wesselink curve, we used the normal points given by Wesselink. The data and their WD fit are shown in Fig. 3.

According to Table 3 in LMD, $E W_{2} / E W_{1} \approx 0.26$ for the lines He I 6678 and 4922 A. When EWs for these lines are calculated using models e.g. by Lanz \& Hubeny (2007), it appears that they are practically constant in the given range of temperatures and are only weakly dependent on $\log g$. Therefore, $L_{2} / L_{1}$ in $V$ should be equal to the observed ratio $E W_{2} / E W_{1}$.

Among the light-curve solutions, only the Wesselink curve gives such a luminosity ratio. Although we would gladly choose the solution of a photoelectric curve (and are not fully satisfied with the quality of our solution of the Wesselink curve because of the not too ideal fit of the minima bottoms) the spectroscopic evidence is clearly more consistent with the Wesselink curve, and is certainly incompatible with the HHH solution. The corresponding parameters of the system are listed in Table 6.
Table 6. Absolute parameters of the eclipsing system.

\begin{tabular}{lcccc}
\hline \hline Parameter & Primary & m.e. & Secondary & m.e. \\
\hline Semiaxis $\left(R_{\odot}\right)$ & 10.4 & 0.1 & 14.4 & 0.1 \\
Mass $\left(M_{\odot}\right)$ & 16.6 & 0.4 & 11.9 & 0.3 \\
Radius $\left(R_{\odot}\right)$ & 9.4 & 0.2 & 5.4 & 0.2 \\
$T_{\text {eff }}(\mathrm{K})$ & 30500 & fixed & 25300 & 740 \\
$\log L / L_{\odot}$ & 4.837 & 0.005 & 4.030 & 0.009 \\
$M_{\text {bol }}$ & -7.34 & 0.04 & -5.33 & 0.05 \\
$\mathrm{BC}(\mathrm{mag})$ & $-2.93^{a}$ & & $-2.51^{b}$ & \\
$M_{\mathrm{v}}$ & -4.41 & 0.04 & -2.82 & 0.05 \\
\hline
\end{tabular}

Notes. ${ }^{(a)}$ Martins et al. (2005). ${ }^{(b)}$ Lanz \& Hubeny (2007).

Since the binary is well detached, we assumed that the evolution of the binary components was not affected by binary interaction effects and can be well compared with evolutionary models for single stars. Using models by Claret (2004), with our values of $R_{1}$ and $T_{1}$ one gets the age $5.9 \mathrm{My}$ and the mass $19.5 M_{\odot}$. The age is close to 5.8 My given by Malysheva (1997, based on uvby and $\mathrm{H} \beta$ photometry) for the cluster NGC 1502 and the mass is explainable by the often observed discrepancy between dynamical and evolutionary masses. In the case of the secondary, Claret's model gives the age $9 \mathrm{My}$ and the mass $11.2 M_{\odot}$ for $R_{2}$ and $T_{2}$. In view of the possible errors, this model is acceptable. A larger $R_{2}$, which would follow from other solutions in Table 5, would infer a yet older age.

The magnitude difference of the third body given by Balega et al. (2007) corresponds to the third body contribution of $29.4 \%$, i.e. agrees well with most of the solutions. Summing up the absolute visual magnitudes of all three components, one derives a total $M_{\mathrm{v}}=-5.02$. With $V_{\max }=6.98$ and $A_{\mathrm{v}}=2.27$ (both numbers according to Crawford 1994), the distance modulus is $m-M=9.73$, close to Crawford's value of 9.71 (Crawford obtained this value as the average moduli of 20 cluster members using uvby and $\mathrm{H} \beta$ photometry analysis).

It is certainly possible to assume synchronized rotation in this close binary. Accepting the solution of the Wesselink curve, the primary and secondary $V_{\text {rot }} \sin i$ velocities should be $172 \mathrm{~km} \mathrm{~s}^{-1}$ and $98 \mathrm{~km} \mathrm{~s}^{-1}$. Using the He I lines $4471 \mathrm{~A}$ and $5876 \mathrm{~A}$ in the ELODIE spectra, we compared their FWHM with tables of Abt et al. (2002) and a formula from Munari \& Tomasella (1999), and derived $V_{\text {rot }} \sin i$ velocities of $160 \mathrm{~km} \mathrm{~s}^{-1}$ for the primary, $92 \mathrm{~km} \mathrm{~s}^{-1}$ for the secondary, and $60 \mathrm{~km} \mathrm{~s}^{-1}$ for the tertiary components, each with an accuracy of about $6 \%$. 
The agreement is not perfect, but better than for other light-curve solutions.

\section{Conclusions}

We have attempted to explain the discrepancy between the $K_{1}$ values of SZ Cam obtained by $\mathrm{HHH}$ and ourselves by the inadequacy of the CCF method for the solution of multiple systems. $\mathrm{HHH}$ also found unrealistic velocities for the third body: absolute values of their third-body radial velocities are larger than $40 \mathrm{~km} \mathrm{~s}^{-1}$, i.e. they disagree with the values given by LMD, Gorda $(2002,2008)$, and this paper. The CCF method produced erroneous results in the case of another binary in the HHH paper, IU Aur: no third light was found, although $l_{3} \approx 30 \%$ was deduced by various ways in this case.

In a broader context, problems with the application of the CCF method were also highlighted by Rucinski (2002). He mentioned namely the unreliable results achieved when the method is applied to three-body systems, and pointed out the uncertainty in the relative luminosity determination - both problems now being illustrated in the case of SZ Cam. In the cases of SZ Cam and IU Aur, the low quality ( $S / N$ of only 40 and 20, respectively) and low number of spectra used by HHH might play a role.

In several papers, the CCF method has been used to study extragalactic binaries. It is rather likely that a third component is often present in these binaries because of spatially unresolved field stars, and the results of the CCF method especially for binaries with light curves of small amplitude might be doubtful.

Acknowledgements. We are obliged to colleagues from the Ondřejov Observatory and their guests who took the SZ Cam and ADS 2984 A spectra (Drs. M. Ceniga, P. Hadrava, A. Kawka, D. Korčáková, L. Kotková, B. Kučerová,
G. Michalska, E. Niemczura, V. Votruba, P. Zasche). P.M. was supported by the Research Project MSM0021620860 of the Ministry of Education, Czech Republic.

\section{References}

Abt, H. A., Levato, H., \& Grosso, M. 2002, ApJ, 573, 359

Balega, I. I., Balega, Yu.Yu., Maximov, A. F., et al. 2007, Astrophys. Bull., 62, 339

Budding, E. 1978, Ap\&SS, 36, 329

Chochol, D. 1980, BAICz, 31, 321

Claret, A. 2004, A\&A, 424, 919

Conti, P., \& Alschuler, W. R. 1971, ApJ, 170, 325

Crawford, D. L. 1994, PASP, 106, 397

ESA 1997, The Hipparcos and Tycho Catalogues (Noordwijk, the Netherlands: ESA), ESA SP-1200

Gorda, S.Yu. 2000, IBVS, 4839

Gorda, S.Yu. 2002, IBVS, 5345

Gorda, S.Yu. 2008, Astrophys. Lett., 34, 1

Gorda, S.Yu., Balega, Yu.Yu., Pluzhnik, E. A., \& Shkhagosheva, Z. U. 2007, Astrophys. Bull., 62, 352

Harries, T. J., Hilditch, R. W., \& Hill, G. 1998, MNRAS, 295, 386 (HHH)

Kitamura, M., \& Yamasaki, A. 1972, Tokyo Astr. Bull., No. 220

Lanz, T., \& Hubeny, I. 2003, ApJS, 146, 417

Lanz, T., \& Hubeny, I. 2007, ApJS, 169, 83

Lorenz, R., Mayer, P., \& Drechsel, H. 1998, A\&A, 332, 909 (LMD) H. 2005, MNRAS, 360, 915

Malysheva, L. K. 1997, PAZh, 23,667

Martins, F., Schaerer, D., \& Hillier, D. J. 2005, A\&A, 436, 1049

Mason, D. R., Gies, D. R., William I. H., et al. 1998, AJ, 115, 821

Massey, P., Puls, J., Pauldrach, A. W. A., et al. 2005, ApJ, 627, 477

Mayer, P., Lorenz, R., Chochol, D., \& Irsmambetova, T. R. 1994, A\&A, 288, L13

Michalska, G., Kubát, J., Korčáková, D., et al. 2007, IAUS, 240, 555

Moultaka, J., Ilovaiski, S. A., Pruguiel, P., \& Soubiran, C. 2004, PASP, 116, 693

Munari, U., \& Tomasella, L. 1999, A\&A, 343, 806

Rucinski, S. M. 2002, AJ, 124, 1746

Wesselink, A. J. 1941, Leiden Ann., 17, No. 3 